\title{
SIS - A TWO STAGE SYNCHROTRON FACILITY FOR HEAVY IONS
}

K. Elasche, D. Böhne, B. Franczak, B. Franzke, C. Riedel

GSI, D 6100 Darmstadt, Postfach 110541 , Fed. Rep. of Germany

\section{Summary}

The GSI studies for a new large $14 \mathrm{GeV} / \mathrm{u}$ heavy ion facility have been continued. Once again concepts for two-stage facilities have been studied that combine a fast cycling booster synchrotron with a slowly cycling high energy synchrotron. In the proposed facility the total power consumption will be moderate, when superconducting magnets are used in the high energy synchrotron, and the operational versatility will allow experiments with free choice of ion species and energy in both energy ranges, 20 to $1000 \mathrm{MeV} / \mathrm{u}$ as well as 1 to $14 \mathrm{GeV} / \mathrm{u}$. With respect to the two-stage concept the design of a new high current linear accelerator has been optimized.

\section{Introduction}

Growing interest in heavy ion physics has stimulated the construction of heavy ion accelerators in the past years. At GSI Darmstadt the Unilac now accelerates all kinds of ions to energies of about $10 \mathrm{MeV} / \mathrm{u}$. This energy range will be extended by insertion of two additional Alvarez cavities up to about $20 \mathrm{MeV} / \mathrm{u}$. In 1979 it was proposed to design a completely new facility for the acceleration of all ions up to uranium in the extremely broad range from $20 \mathrm{MeV} / \mathrm{u}$ to $14 \mathrm{GeV} / \mathrm{u} .{ }^{1}$ at present the Bevalac in Berkeley and the Synchrophasotron in Dubna provide light particles with energies to 2.2 GeV/u respectively $3.5 \mathrm{GeV} / \mathrm{u}$. The GSI project for a beavy ion synchrotron (SIS) will open new experimental possibilities to investigate the yet unexplored energy range (Fig. 1). For the $14 \mathrm{GeV} / \mathrm{l}$ facility two different concepts were discussed:

(a) a large rather fast cycling synchrotron (SIS 100) that accelerates all heavy ions in the energy range from $20 \mathrm{MeV} / \mathrm{u}$ to $14 \mathrm{GeV} / \mathrm{u}$ and

(b) a two-stage machine with a small rapid cycling booster synchrotron for energies to at least $1 \mathrm{GeV} / \mathrm{u}$ and a large slowly cycling synchrotron which might be built with superconducting magnets.

At first the single stage concept was studied thoroughly as the shortest way to relativistic heavy ion beams. ${ }^{2}$ In 1980 once again two-stage concepts have come under study which provide a reduction in total power consumption and the operational versatility of two stages.

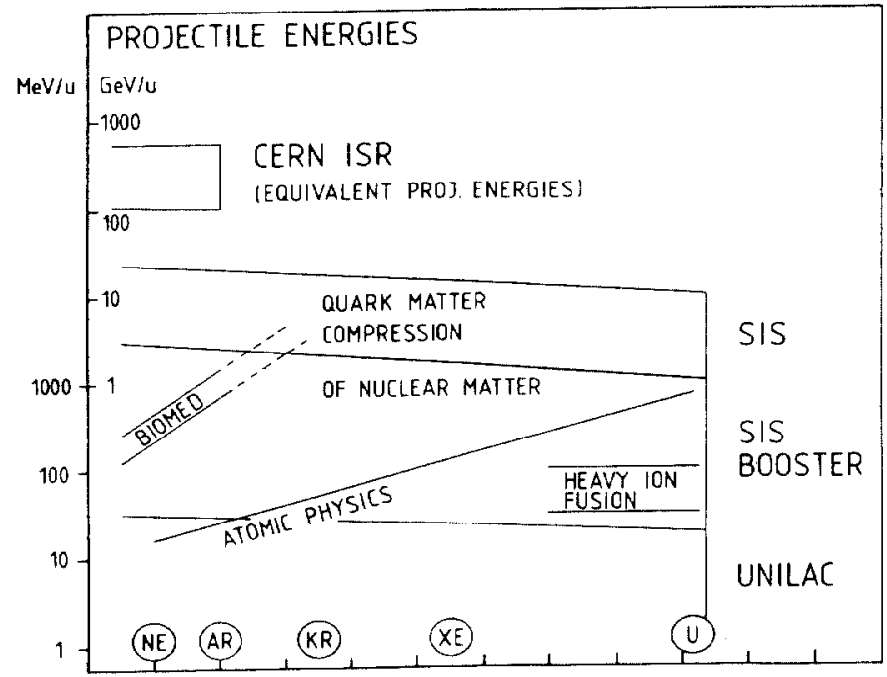

Fig.1: Energy range for SIS and fields of scientific interest

\section{The two-stage synchrotron facility}

Fig.2 presents a survey of two different schemes for a two-stage facility at GSI. In general, two synchrotrons will offer the following advantages when compared to one large synchrotron like SIS 100, namely:

(a) Two separate accelerators are available which could be used quasi-independently, as shown in the operation mode of Fig. 3. In both

- the medium energy range (20 MeV/u up to about 1 $\mathrm{GeV} / \mathrm{u}$ ) and

- the high energy range ( $1 \mathrm{GeV} / \mathrm{u}$ up to $15 \mathrm{GeV} / \mathrm{u}$ )

free choice of ion energy and ion species is provided.

(b) The flexibility of a two-stage facility opens new roads for experimental programs such as

- storage of highly stripped ions in the main ring,

- deceleration of the stored highly stripped ion beams in the first ring for experiments at low energies,

- experiments at high duty cycles (up to 100\%) for the medium energy range with the supereonducting main ring operating as stretcher ring.

(c) The large high energy synchrotron will be operated at low repetition rates. Nevertheless, by the use of a booster synchrotron the slowly cycling main ring could yield higher particle currents than the fast cycling single stage SIS 100 (Fig. 4). A low ramping rate ( $\mathrm{dB} / \mathrm{dt} \leq 0.5 \mathrm{~T} / \mathrm{s})$ strongly facilitates development and operation of superconducting synchrotron magnets.

(d) Many technical systems in the large main ring will become simpler and less expensive by injection at high energies using a booster synchrotron, e.g.

- the $\mathrm{rf}$ accelerating system (reduction of frequency range and number of cavities),

- the vacuum system $\left(\leq 1 \times 10^{-9}\right.$ Torr instead of $1 \times$ $10^{-11}$ Torr)

- the main power supplies (active power during field ramping).

Even more important is the reduction in total power consumption for a two-stage facility as compared to the single stage SIS 100 that could be achieved with slowly cycling superconducting magnets in the main $\mathrm{ring}$.

on the other hand, compared to the SIS 100 a two-stage facility will require

(a) a longer construction time for the complete facility,

(b) more man power for the operation of both machines, and

(c) additional research and development efforts for the construction of reliable superconducting synchrotron magnets.

The time schedule for the construction of a two-stage facility will strongly depend on the progress made in the development of superconducting synchrotron magnets for the main ring. With a cautious design of $4 \mathrm{~T}$ for the maximum field the main ring would consist of 80 magnets each $2 \mathrm{~m}$ long. Both maximum field and magnet length are moderate compared to the design for Tevatron $(\mathrm{E}=4.4 \mathrm{~T}, \mathrm{~L}=6.12 \mathrm{~m})$, Isabelle $(\mathrm{B}=5 \mathrm{~T}, \mathrm{~L}=4.75 \mathrm{~m})$ and HERA ( $B=4.7 \mathrm{~T}, L=5.8 \mathrm{~m}$ ). It might be even more attractive to make use of new $8 \mathrm{~T}$ synchrotron magnets, since these high field magnets will be developed for the next generation of large proton machines. The diameter of a $15 \mathrm{GeV} / \mathrm{u}$ main ring would shrink to about $70 \mathrm{~m}$ with $8 \mathrm{~T}$ magnets.

The maximum energy for the booster synchrotron has to 

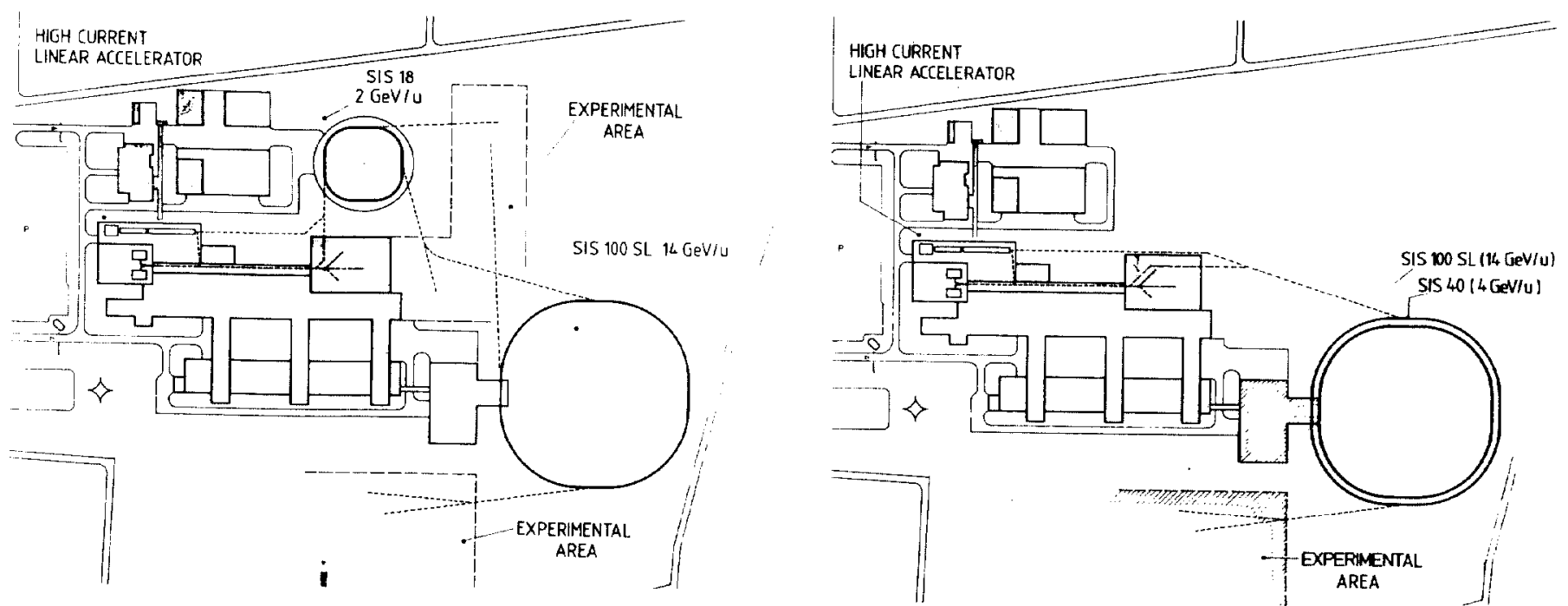

Fig.2: GSI site plans for two-stage $15 \mathrm{GeV} / \mathrm{u}$ heavy ion facilities

be chosen with respect to a list of arguments. An energy of at least $0.5 \mathrm{GeV} / \mathrm{u}$ is required for the production of fully stripped uranium ions. Therefore, a few years ago it was proposed to construct a small 50 m diameter machine with maximum energies of $0.8 \mathrm{GeV} / \mathrm{u}$ for uranium and $2 \mathrm{GeV} / \mathrm{u}$ for light ions (Fig. 2a). Such a small booster synchrotron (SIS 18) would be adequate for a broad experimental program of nuclear physics, atomic physics, and biomedical and technical applications. On the other side, there are arguments for higher energies of the booster synchrotron:

(a) In the energy range from 2 to $5 \mathrm{GeV} / \mathrm{u}$ interesting phenomena due to the compression of nuclear matter are expected. Since interest in these experiments has grown strongly, one would like to proceed as soon as possible into the unexplored energy range.

(b) If interest in experiments with heavy ions for elementary particle studies should further develop,
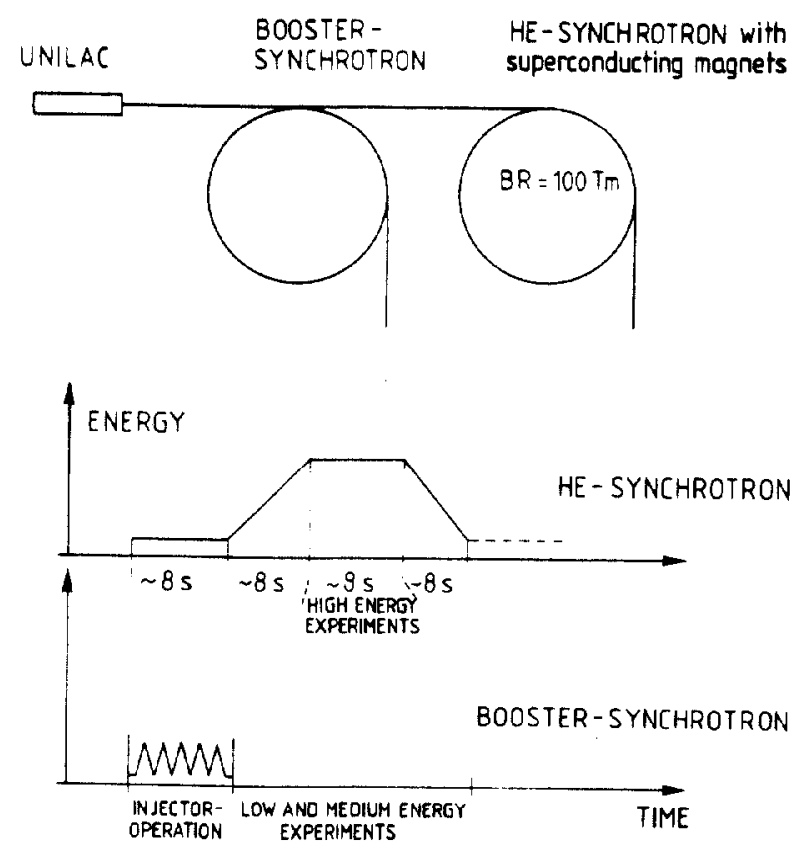

Fig.3 Operation mode for a two-stage facility with fast-cycling booster synchrotron and slowly cycling main ring intersecting storage rings could provide the necessary high center of mass energies. In that case a booster synchrotron with maximum energies of 3-4 $\mathrm{GeV} / \mathrm{u}$ could serve as an appropriate injector machine, whereas injection at low energies between 1 and $2 \mathrm{GeV} / \mathrm{u}$ would restrict particle currents and hence luminosities in the storage ring facility due to space charge effects at injection.

Therefore, a larger booster synchrotron (SIS 40) has also been studied that might have the same diameter as the main ring (Fig.2b).

At present the Unilac cannot provide the particle currents that are needed to fill the booster synchrotron to the incoherent space charge limit. In order to provide particle currents in the range of $10 \mathrm{~mA}$, reflex ion sources for singly or doubly charged ions and a new high current linear accelerator with a duty cycle of about 1 were proposed (Fig.5).

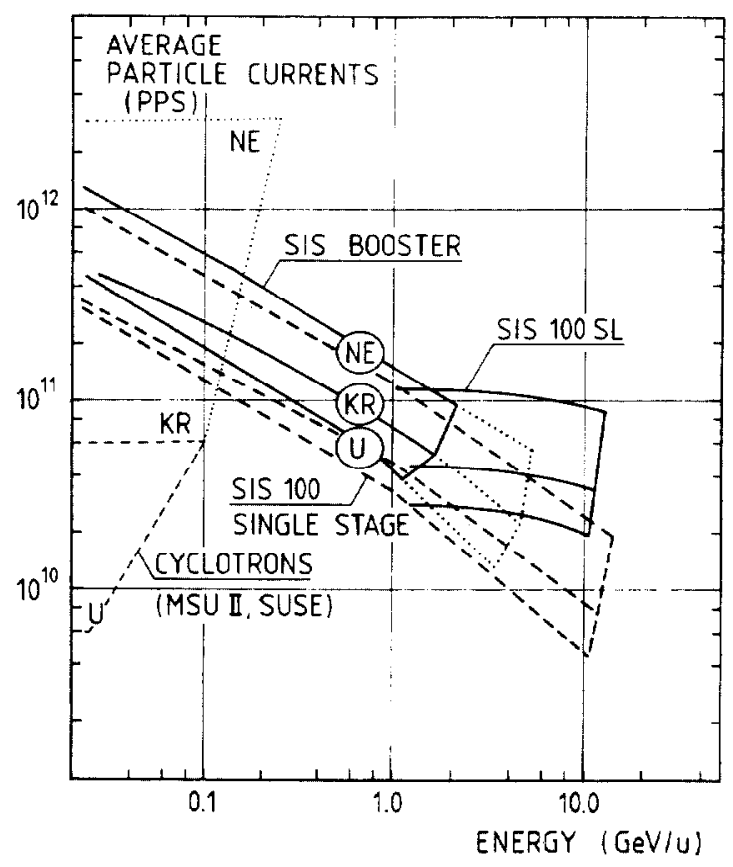

Fig. 4 Average particle current versus energy for a two stage facility compared to the output of the single stage SIS 100 


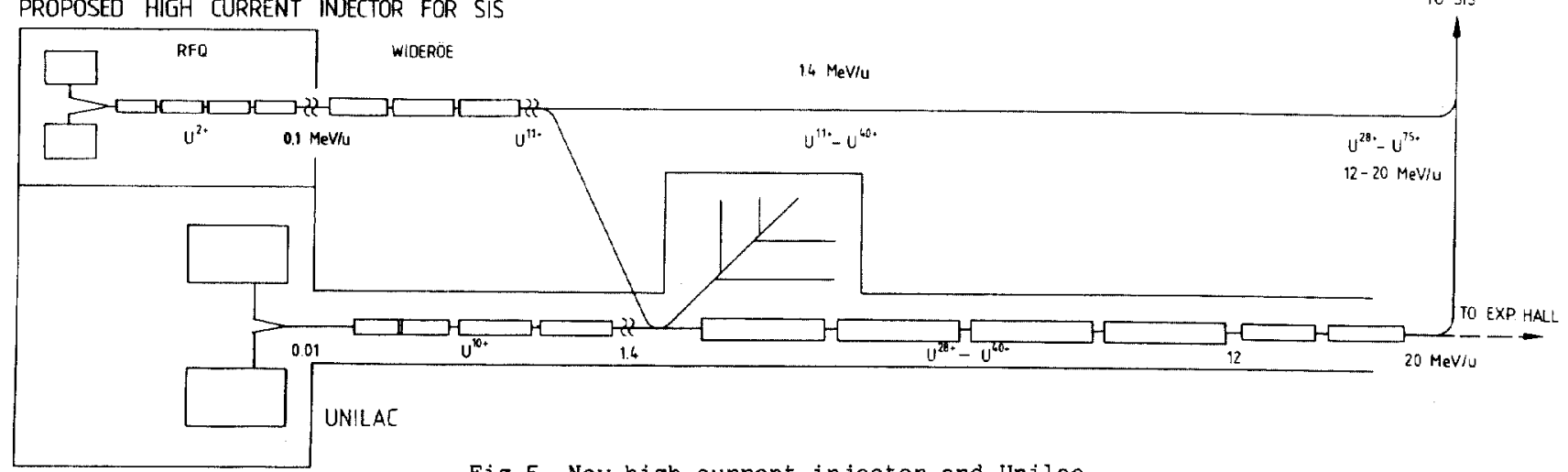

Fig.5 New high current injector and Unilac

\section{Design parameters tor two-stage facilities}

\section{Magnets}

For the booster synchrotron conventional magnets will be preferred in order to achieve repetition frequencies of about $1 \mathrm{~Hz}$. Especially for a small booster synchrotron like SIS 18 , the power consumption of the magnet system is moderate. For the main ring, however, superconducting synchrotron magnets are proposed, since in this stage long cycle times around $30 \mathrm{~s}$ do not reduce the average particle currents remarkably. The power consumption of the superconducting magnet system, which is the sum of losses in the power supplies, cabling, and mainly the refrigerator losses, is also moderate. Hence, the total power consumption of a two-stage facility, especially with a small booster synchrotron, is smaller than the consumption of the single stage SIS100.

$$
\text { SIS } 10 \text { SIS } 40 \text { SIS } 100 \mathrm{SL} \text { SIS100 }
$$

$\begin{array}{lcccc}\text { Machine diameter (m) } & 52 & 140 & 140 & 250 \\ \begin{array}{l}\text { Number and length (m) } \\ \quad \text { of dipole magnets }\end{array} & 48(1.3) & 56(2.6) & 56(2.6) & 128(2.7) \\ \text { Ramping rate (T/s) } & 10 & 4 & 0.5 & 2 \\ \begin{array}{l}\text { Power consumption } \\ \quad \text { for magnets and }\end{array} & 1 & 3 & 2 & 8 \\ \quad \text { cryogenic cooling (Mw) } & & & \end{array}$

Tab. 1 Synchrotron magnets and power consumption

RF accelerating cavilies

In the single stage SIS 100 at least $12 \mathrm{rf}$-cavities are required, since the total of accelerating voltage, which is proportional to the ramping rate $d B / d t$, to the bending radius, and to the circumference amounts to $135 \mathrm{kV}$ per turn. In a two-stage facility, the main ring with superconducting magnets is smaller and is operated at a low ramping rate and, therefore, needs only an accelerating voltage of $15 \mathrm{kV}$; the required accelerating voltage for the booster synchrotron strongly depends on the machine diameter. Anyhow, the total costs for the $r f$ accelerating system will be reduced in a two-stage facility.

SIS 18 SIS 40 SIS 100 SL SIS 100

\begin{tabular}{lcccc}
\hline Number & 2 & 8 & 2 & 12 \\
$\begin{array}{l}\text { Accelerating } \\
\text { voltage } \mathrm{U}_{\mathrm{S}}(\mathrm{kV})\end{array}$ & 35 & 130 & 15 & 185 \\
$\begin{array}{c}\text { Frequeney } \\
\text { range (MHz) }\end{array}$ & $0.8-8$ & $0.8-8$ & $4-8$ & \\
Costs (MDM) & 2.5 & 10 & 1.5 & 15
\end{tabular}

Tab.2 RF accelerating sections

\section{Vacuum system}

Particle losses in the circulating beam due to charge changing collisions with the residual gas in the vacuum chamber mainly occur in the energy range up to 100 $\mathrm{MeV} / \mathrm{u}$. Therefore, the operating pressure in the booster-synchrotron has to be $1 \times 10^{-11}$ Torr just like in the single stage SIS 100 . In the main ring an operating pressure of $1 \times 10^{-9}$ Torr seems adequate due to the injection of fully ionized projectiles at high energy. However, it might be useful to operate the main ring at lower pressure with respect to the pressure bump effect and the stretcher ring mode.

SIS 18 SIS 40 SIS 100 SL SIS 100

$\begin{array}{lcccc}\text { Operating pressure } & & & & \\ \quad \text { (Torr) } & 1 \times 10^{-11} & 1 \times 10^{-11} & \leq 1 \times 10^{-9} & 1 \times 10^{-11} \\ \text { Circumference (m) } & 160 & 450 & 450 & 780 \\ \text { Costs (MDM) } & 3.5 & 5 & 4.5 & 8\end{array}$

Tab. 3 Parameters for the vacuum-system

\section{Project status and schedules}

At present research and development concentrates on two main points: (a) high current ion sources and RFQ-structures for a new high current linac and (b) magnets, accelerating cavities, and ultra high vacuum systems as major components of a heavy ion synchrotron. Tab.4 shows the estimated costs as well as time and personnel schedules. The costs for a high current linear accelerator and experimental facilities were omitted. Also research and development for superconducting synchrotron magnets is not included, since detailed studies are required for reliable estimates.

SIS 18 SIS 10OSL COMPLETE SIS 100 FACILITY

$\begin{array}{lrrrr}\text { Costs (MDM) } & 48 & 72 & 120 & 110 \\ \text { for synchrotrons } & & & & \\ \text { construction time } & 4 & 4-5 & 8-9 & 5-6 \\ \text { Personnel } & 45 & 45 & 90 & 64\end{array}$

Tab. 4 Cost estimates and schedules

\section{References}

1 SIS - A Relativistic Heavy Ion Accelerator Facility, GSI Study Report, May 1979

2 SIS - An Accelerator Facility for Relativistic Heavy Ions, $11^{\text {th }}$ International Conference on High-Energy Accelerators, CERN 1980, 220. 